\title{
OTR3 - Validação de ensaio imunoenzimático (Western blot) para o diagnóstico da histoplasmose
}

Marcos de Abreu Almeida ${ }^{1 *}$; Cláudia Vera Pizzini ${ }^{1}$; Regina Helena Saramago Peralta ${ }^{2}$; José Mauro Peralta ${ }^{3}$; Raquel de Vasconcelos Carvalhaes Oliveira ${ }^{1}$; Carla Lourenço Tavares de Andrade ${ }^{4}$; Rosely Maria Zancopé Oliveira ${ }^{1}$.

\section{1 - INI/Fiocruz;}

2 - Universidade Federal Fluminense;

3 - Universidade Federal do Rio de Janeiro;

4 - ENSP/Fiocruz.

\section{Introdução:}

A histoplasmose é uma micose cosmopolita causada pelo fungo dimórfico Histoplasma capsulatum, cujo habitat é o solo rico em excretas de aves e morcegos. Esta infecção ocorre a partir da inalação de propágulos de $H$. capsulatum e apresenta um amplo espectro clínico, variando de formas leves a disseminadas, a depender do inóculo infectante, do status imunológico do hospedeiro e da virulência da cepa. O diagnóstico da histoplasmose é baseado em aspectos clínicos, epidemiológicos, radiológicos e laboratoriais, embora alguns sintomas possam ser confundidos com outras doenças, tais como a tuberculose e outras micoses. O teste de referência para a confirmação do diagnóstico é o isolamento e identificação de H. capsulatum em cultivo. Entretanto, na ausência dos mesmos, a sorologia tem sido utilizada para o diagnóstico presuntivo da histoplasmose através da detecção de anticorpos. O principal complexo antigênico utilizado para a detecção de anticorpos anti- $H$. capsulatum é a histoplasmina, constituída principalmente pelos antígenos C, H e M. Estes últimos, em sua forma nativa, são glicoproteínas contendo epítopos protéicos específicos e glicosídicos inespecíficos. Deglicosilações químicas pelo metaperiodato de sódio (NaIO4) provaram aumentar a sensibilidade e especificidadeem métodos imunoenzimáticos, tais como Western blot e ELISA, reduzindo a reatividade cruzada com outros fungos.

\section{Objetivo:}

O principal objetivo do presente estudo foivalidar o método imunoenzimático (Western blot) para a detecção de anticorpos no diagnóstico da histoplasmose. 


\section{Metodologia:}

Desta forma, foi realizado um estudo casocontrole, utilizando 118 amostras de soro de pacientes com histoplasmose e 118 amostras de soro de indivíduos com história clínico-epidemiológica compatível com micose sistêmica, saudáveis ou acometidos por outra micose ou tuberculose, residentes no estado do Rio de Janeiro. As amostras foram coletadas no período de 2000 a 2013 e encaminhadas ao Laboratório de Micologia do Instituto Nacional de Infectologia Evandro Chagas. Os parâmetros de validação diagnóstica foram calculados considerando a categorização dos resultados obtidos em uma tabela 2 X 2 e analisados pelo SPSS 17.0.

\section{Resultados:}

O Western blot demonstrou uma sensibilidade de 94,9\%, especificidade de $94,1 \%$, acurácia de $94,5 \%$ e uma precisão quase perfeita. As fitas demonstraram-se viáveis para utilização por até cinco anos após a sensibilização com o antígeno HMIN-PT. Estes resultados comprovam a validade do ensaio imunoenzimático (Western blot) para detecção de anticorpos anti-H. capsulatum utilizando HMIN-PT como uma ferramenta de boa acurácia no diagnóstico da histoplasmose e reprodutível.

\section{Conclusão:}

O ensaio imunoenzimático Western blot contribuirá para o melhoramento do diagnóstico desta micose, uma vez que esta técnica permitirá a obtenção de resultados em menos de 24 horas, o que constitui um grande avanço em relação às técnicas existentes na atualidade e poderá vir a ser disponibilizada em outros centros do Sistema Único de Saúde.

\section{Palavras-Chave: Histoplasmose, Imunodiagnóstico e Western blot}

